\title{
Propagation of Picosecond Pulses on Superconducting Transmission Line Interconnects
}

\author{
Vladimir V. Talanov ${ }^{1}$, Derek Knee ${ }^{2}$, David Harms ${ }^{1}$, Kieran Perkins ${ }^{1}$, \\ Andrew Urbanas ${ }^{1}$, Jonathan Egan ${ }^{1}$, Quentin Herr ${ }^{1}$, and Anna Herr ${ }^{1}$ \\ ${ }^{1}$ Northrop Grumman Corp., Baltimore, MD 21240 and \\ ${ }^{2}$ Microsoft Research, Redmond, WA 9805为
}

(Dated: 14 April 2021)

\begin{abstract}
Interconnects are a major discriminator for superconducting digital technology, enabling energyefficient data transfer and high-bandwidth heterogeneous integration. We report a method to simulate propagation of picosecond pulses in superconducting passive transmission lines (PTLs). A frequency-domain propagator model obtained from the Ansys High Frequency Structure Simulator (HFSS) field solver is incorporated in a Cadence Spectre circuit model, so that the particular PTL geometry can be simulated in the time-domain. The Mattis-Bardeen complex conductivity of the superconductor is encoded in the HFSS field solver as a complex-conductivity insulator. Experimental and simulation results show that $\mathrm{Nb} 20 \Omega$ microstrip PTLs with $1 \mu \mathrm{m}$ width can support propagation of a single-flux-quantum pulse up to $7 \mathrm{~mm}$ and a double-flux-quantum pulse up to $28 \mathrm{~mm}$.
\end{abstract}

Low-loss interconnects are a major advantage for superconducting digital technology as they support serial data links at the on-chip clock rate and with the on-chip signal levels. This enables off-die communication with low overhead [1]- 3]. The transmission energy in superconducting passive transmission lines (PTLs) links is on order fJ/bit including the $300 \times$ cooling overhead. This is four orders of magnitude lower than energy-efficient CMOS interconnects reporting $0.5-2 \mathrm{pJ} /$ bit inter-chiplet communication [4] and 0.1-1 nJ/bit for server-to-server communication 5]. Superconducting links share the essential properties of optical interconnect but without the overhead of low conversion efficiency.

Starting from the demonstration of $60 \mathrm{~Gb} / \mathrm{s}$ chip-tochip communication, superconducting off-die PTL links have advanced to demonstration of 16 -bit buses [3] using a single chip on an multi-chip module (MCM), and the demonstration of an MCM with multiple chips of different sizes and with synchronous communication between them [6]. This design used an accurate model for the PTL interconnects as presented in this paper, enabling simulation and verification methods compatible with industry standard CAD tools.

Accurate PTL modeling must capture the attenuation and dispersion of signals with analog bandwidth extending to sub-THz. The energy efficient superconducting logic families [7], [8] encode data in a form of single-fluxquantum (SFQ) pulses of picosecond duration. Only quantum flux parametrons (QFPs) are incompatible with PTL interconnect, requiring SFQ signal translation for transmit and receive [9].

The SFQ pulse spectrum, $360 \mathrm{GHz}$ for Josephson junc-

\footnotetext{
* This research is based upon work supported in part by the ODNI, IARPA, via ARO. The views and conclusions contained herein are those of the authors and should not be interpreted as necessarily representing the official policies or endorsements, either expressed or implied, of the ODNI, IARPA, or the U.S. Government.
}

tions with critical current density of $100 \mu \mathrm{A} / \mu \mathrm{m}^{2}$, approaches the material energy-gap frequency of $720 \mathrm{GHz}$ for $\mathrm{Nb}$ interconnect. Previous simulation studies have modeled the data links using a two-fluid model [10], [11]. The model is applicable up to $100 \mathrm{GHz}$, for PTLs with dielectric that is thick compared to the magnetic penetration depth, $\lambda$. Short-range propagation of SFQ pulses on PTLs has been confirmed in numerous experiments, e.g. [12]-15] but accurate modeling is required for future systems with sub-micron, high-impedance PTLs covering distances up to 1 meter.

Kautz was the first to accurately model the propagation of picosecond pulses on superconducting transmission line with a numerical approach incorporating the Mattis-Bardeen microscopic theory [16] for the superconductor complex conductivity $\sigma=\sigma_{1}-i \sigma_{2}$ 17. Shortly after Peterson and McDonald reported the generation of such pulses in the Josephson junction [18], Kautz considered a parallel-plate transmission line with $\mathrm{Nb}$ groundplane and $\mathrm{Pb}$-In stripline, and showed that dispersion both attenuates and distorts the pulse while it propagates. Chi et al. verified Kautz's result qualitatively by electro-optic sampling of picosecond pulses in a Nb coplanar transmission line [19], and comparing to the model.

Kautz's approach applies to a wide microstrip PTL where the fringe-fields can be ignored. Real PTLs, however, may include any number of complications, as they may be microstrips whose width is comparable to the dielectric thickness, may be coplanar or stripline, may be in proximity to via walls or other transmission lines, may utilize an insulator with non-uniform dielectric constant, and may incorporate discontinuities such as bends, vias, or crossings [10]. A 3D field solver such as Ansys HFSS [20] allows accurate modeling of all of this, provided an accurate frequency-dependent model of the dispersive material.

Here we report a method to simulate propagation of picosecond pulses in superconducting PTLs with arbitrary length and geometry. The method uses a frequency domain propagator model obtained from the Ansys HFSS 
field solver to produce a Cadence Spectre circuit model, for time-domain simulation. In the frequency-domain model, the scattering matrix $\hat{S}(\omega)$ between input and output ports of the PTL is obtained using the MattisBardeen complex conductivity. $\hat{S}(\omega)$ is converted into the impulse response matrix $\hat{S}(t)$ to produce the timedomain model, implemented in Verilog-A. This model is used to simulate and optimize the PTL link including the reciprocal quantum logic (RQL) driver and receiver. The models are verified by comparing to the known theoretical solutions for a wide microstrip [17] and by comparing to experimental data.

\section{GENERAL METHOD}

The frequency-domain and time-domain responses of any multiport network can be related by [21]

$$
\hat{b}(\omega)=\hat{a}(\omega) \hat{S}(\omega), \quad \hat{b}(t)=\int_{-\infty}^{\infty} \hat{a}(\tau) \hat{S}(t-\tau) d \tau
$$

where $\hat{a}(\omega)$ and $\hat{a}(\tau)$ refer to the incident wave, $\hat{b}(\omega)$ and $\hat{b}(t)$ refer to the reflected wave, $\hat{S}(\omega)$ refers to the scattering matrix, and $\hat{S}(t)$ refers to the impulse-response matrix. Each pair is related via the Fourier transforms. In a quality model, $\hat{S}(\omega)$ must be reciprocal, passive, causal, have sampling density adequate to resolve all features, and be defined over the entire spectrum of interest [22, 23].

We apply the above method to a PTL with length $L$ and transmission coefficient $S_{21}(\omega)=e^{-\gamma L}$, where $e^{-\gamma L}$ is the propagation factor, $\gamma(\omega)=\alpha+i \beta$ is the propagation constant, $\alpha$ is the attenuation constant, and $\beta$ is the phase constant. The PTL input and output voltage in the frequency domain relate as

$$
V_{\text {out }}(\omega)=V_{\text {in }}(\omega) e^{-\gamma L}
$$

The Fourier transform of the input transient voltage $V_{\text {in }}(t)$ is $V_{\text {in }}(\omega)$; the inverse Fourier transform of $V_{\text {out }}(\omega)$ yields the output transient $V_{\text {out }}(t)$.

To implement the time-domain model, the PTL propagation factor is used to construct an S-parameter file to be used in the Spectre time-domain circuit simulation. The full-length S-parameter data is calculated during netlisting and is given as an input to a Verilog-A wrapper. The model incorporates Spectre's "nport" device that provides the impulse response. Spectre performs the inverse Fourier transform of the scattering matrix

$$
\hat{S}_{\mathrm{PTL}}(\omega)=\left[\begin{array}{cc}
0 & 0 \\
e^{-\gamma L} & 0
\end{array}\right]
$$

to create the impulse response matrix $\hat{S}_{\mathrm{PTL}}(t)$. The output transient is

$$
V_{\text {out }}(t)=\int_{-\infty}^{\infty} V_{\text {in }}(\tau) \hat{S}_{\mathrm{PTL}}(t-\tau) d \tau
$$

which is the convolution of the impulse response with the input signal, performed during the transient analysis.

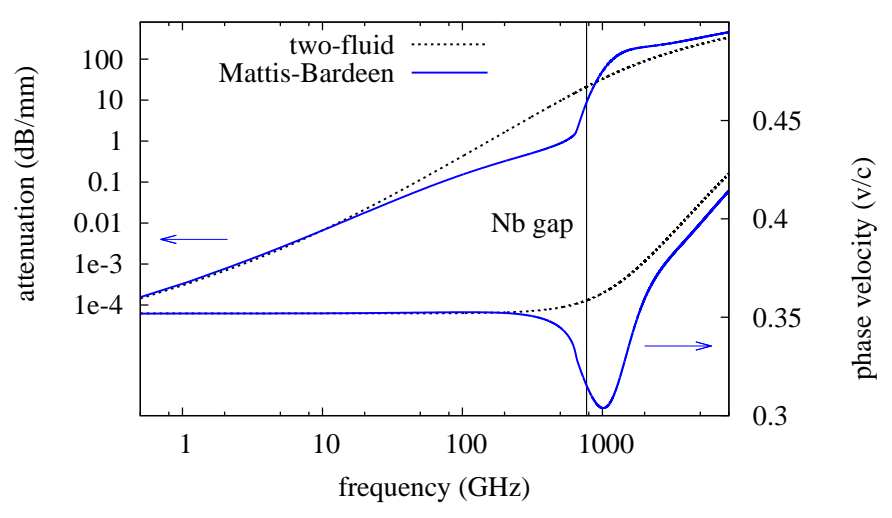

FIG. 1. Comparison of the frequency-dependent attenuation and phase velocity between Mattis-Bardeen and the two-fluid model simulated for $\mathrm{Nb}$ wide microstrip line. Phase velocity is normalized to the speed of light, $c$. The models are aligned at $10 \mathrm{GHz}$.

\section{FREQUENCY DOMAIN MODEL AND SIMULATIONS}

To produce an analytical reference, we first calculate the complex propagation constant $\gamma=\alpha+i \beta$ of a wide microstrip formed by identical superconducting plates following the approach in 24]:

$$
\begin{gathered}
\beta=\omega \sqrt{\varepsilon_{0} \varepsilon_{r} \mu_{0}} \sqrt{1+\frac{2\left(X_{\mathrm{eff}}-R_{\mathrm{eff}} \tan \delta\right)}{\omega \mu_{0} s}} \\
\alpha=\frac{\beta}{2}\left[\frac{2 R_{\mathrm{eff}}}{\omega \mu_{0} s+2\left(X_{\mathrm{eff}}-R_{\mathrm{eff}} \tan \delta\right)}+\tan \delta\right]
\end{gathered}
$$

where $\mu_{0}$ is the vacuum permeability, $s$ is the dielectric thickness, $\tan \delta$ is the dielectric loss tangent, $\varepsilon_{0}$ is the vacuum permittivity, $\varepsilon_{r}$ is the interlayer dielectric constant, and it is assumed that $\alpha<<\beta$. $R_{\text {eff }}$ and $X_{\text {eff }}$ are the real and imaginary parts of the effective surface impedance of the superconducting plate of thickness $d$ [17] 25]

$$
Z_{\mathrm{eff}}=R_{\mathrm{eff}}+i X_{\mathrm{eff}}=\sqrt{i \mu_{0} \omega / \sigma} \operatorname{coth}\left(\sqrt{i \mu_{0} \omega \sigma} d\right)
$$

To simulate the PTLs in HFSS we use the MattisBardeen microscopic theory [16] for the real and imaginary parts of $\mathrm{Nb}$ complex conductivity $\sigma=\sigma_{1}-i \sigma_{2}$. Fig. 1 shows the frequency-dependent attenuation and phase velocity of Mattis-Bardeen model compared to the two-fluid model. The two-fluid model overestimates attenuation and slightly underestimates dispersion at frequencies approaching the gap. Overall, Mattis-Bardeen predicts further propagation distance for the SFQ pulses. In the current process the spectral width of the SFQ pulse is about $350 \mathrm{GHz}$ so we expect the two-fluid quadratic dependence of propagation distance on pulse width. In an advanced process with higher critical-current-density 
a)

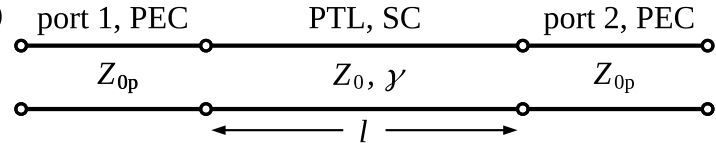

b)

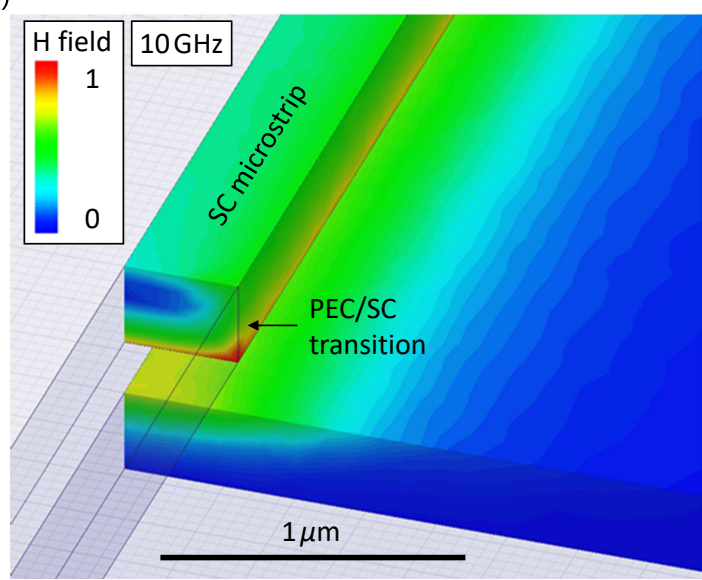

FIG. 2. a) Schematic and b) HFSS model for a $1 \mu \mathrm{m}$ wide superconducting PTL. The simulation is in half-space, exploiting bilaterial symmetry. The length $l$ is $100-200 \mu \mathrm{m}$. The magnetic field intensity in the superconductor PTL is shown at the port-PTL interface. The impedance mismatch between the PEC port with characteristic impedance $Z_{0 p}$ and the superconductor PTL with characteristic impedance $Z_{0}$ causes a slight non-uniformity in the magnetic field near the portPTL interface. In this simulation Nb thickness is $200 \mathrm{~nm}$ and dielectric thickness is $150 \mathrm{~nm}$. A dielectric material model with $\varepsilon_{r}$ of 4.2 and $\tan \delta$ of $10^{-3}$ are representative of the $\mathrm{SiO}_{2}$ interlayer dielectric used in superconducting processes [26].

junctions the difference between the two models will be more significant.

In HFSS, the superconducting metal has been modeled as a material with bulk conductivity $\sigma_{1}$ and negative relative permittivity $-\sigma_{2} / \varepsilon_{0} \omega$. This model is based on the general relationship between the complex permittivity and complex conductivity of any material as $\varepsilon=\varepsilon_{0}\left(\varepsilon_{1}-i \varepsilon_{2}\right)=-i \sigma / \omega$, where $\varepsilon_{1}=-\sigma_{2} / \varepsilon_{0} \omega$ and $\varepsilon_{2}=\sigma_{1} / \varepsilon_{0} \omega$ are the real and imaginary parts of the relative permittivity. While we have modeled the superconductor as having real bulk conductivity and negative relative permittivity, the latest version of HFSS allows for a more direct approach by defining materials with complex bulk conductivity.

Mattis-Bardeen theory offers no analytical expressions for the superconductor complex conductivity, so we use HFSS datasets for piece-wise representation of Nb material parameters. We employ the theory to tabulate the $\mathrm{Nb}$ bulk conductivity and relative permittivity vs. frequency for 1-1000 GHz at 1000 frequency points, and import the datasets into HFSS. The Mattis-Bardeen theory parameters which are superconductor normal conductivity and energy gap, are chosen to give the penetration $\operatorname{depth} \lambda=\operatorname{Im}\left[\sqrt{i \mu_{0} \omega / \sigma}\right] / \mu_{0} \omega=90 \mathrm{~nm}$ and to give the

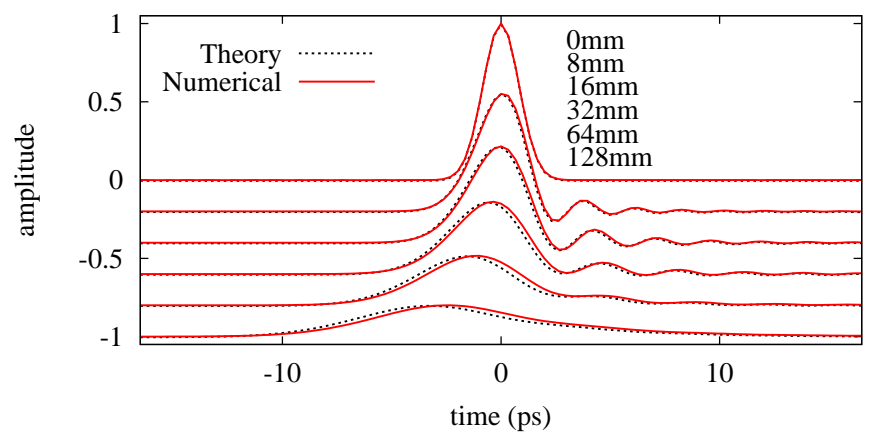

FIG. 3. Pulse propagation on a superconducting passive transmission line. Subsequent traces are offset by $-0.2 \mathrm{mV}$ for legibility. "Theory" is an analytical solution using Eq. (1-2), and "Numerical" is HFSS simulation. Geometrical and material parameters are similar except for PTL width. A $4 \mu \mathrm{m}$ width in HFSS simulations was chosen to produce a small fringe-field effect, giving good agreement with the analytical theory.

intrinsic surface resistance $R_{s}=\operatorname{Re}\left[\sqrt{i \mu_{0} \omega / \sigma}\right]=20 \mu \Omega$ at $10 \mathrm{GHz}$ and $4.2 \mathrm{~K}$. These numbers are representative of $\mathrm{Nb}$ interconnect with $T_{c}$ of $9 \mathrm{~K}$.

Fig. 2 shows a schematic and HFSS 3D model for a PTL terminated with Perfect Conductor (PEC) ports of the same cross-sectional geometry, as HFSS does not allow superconductor ports. The perfect-conductor model has zero resistance and zero kinetic inductance, so current flows only on the surface. After deembedding the ports, the propagation factor for the PTL is computed as

$$
e^{-\gamma l}=\frac{1-S_{11}^{2}+S_{21}^{2} \pm \sqrt{\left(1-S_{11}^{2}+S_{21}^{2}\right)^{2}-4 S_{21}^{2}}}{2 S_{21}}
$$

where $S_{i j}$ are the elements of the scattering matrix found by the HFSS solver, and $l$ is the length of the simulated PTL. A deriviation can be found in the Appendix. The requirement that the real and imaginary parts of the propagation constant be positive defines the sign of the square root. It is tractable for simulations to calculate 100-200 points for the propagation factor for frequencies of $1-1000 \mathrm{GHz}$.

The HFSS engine limits the aspect ratio of any feature to about 10,000:1, which limits the PTL model to a few $\mathrm{mm}$ in length. To extend the model to arbitrary length, $L$, we mathematically construct $e^{-\gamma L}$ from the simulated propagation factor $e^{-\gamma l}$ simulated for the PTL of length $l$. Implementation details of our procedure include: 1) The function $\operatorname{Arg}\left[e^{-\gamma l}\right]$ returns the phase angle in the range $\pm \pi$. The discontinuities are removed to recover true continuous phase using e.g. MATLAB's "unwrap" function. 2) A virtual point at zero-frequency is added with appropriate phase and amplitude corresponding to zero attenuation. 3) Both magnitude and continuous phase are interpolated between the simulated frequency points us- 


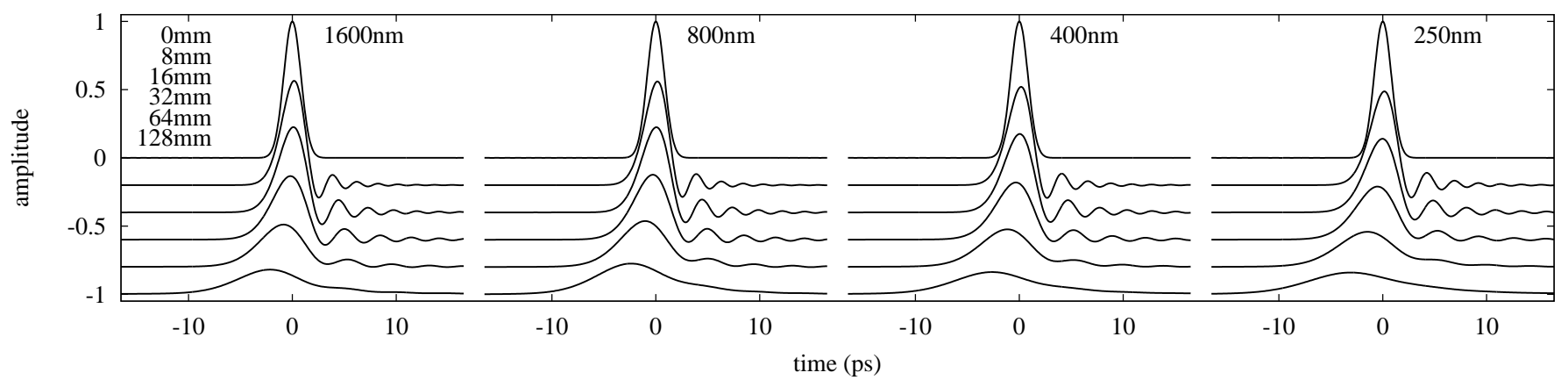

FIG. 4. HFSS simulation of SFQ pulse propagation on a superconducting PTL for four microstrip widths, each at six lengths. Subsequent traces are again offset by $-0.2 \mathrm{mV}$ for legibility. Dielectric thickness is $200 \mathrm{~nm}$, Nb microstrip and ground plane thickness is $300 \mathrm{~nm}$, the dielectric constant is $5.65, \tan \delta$ is 0.0005 , the Nb intrinsic surface resistance, $R_{s}$, is $20 \mu \Omega$ at $10 \mathrm{GHz}$, and the penetration depth $\lambda$ is $90 \mathrm{~nm}$.

ing piece-wise functions $A(\omega)$ and $\theta(\omega)$ :

$$
\left|e^{-\gamma l}\right| \rightarrow A(\omega), \quad \operatorname{unwrap}\left(\operatorname{Arg}\left[e^{-\gamma l}\right]\right) \rightarrow \theta(\omega)
$$

where the arrows denote the interpolation. Finally, we compute the full-length propagation factor

$$
e^{-\gamma(\omega) L}=A(\omega)^{L / l} e^{i \theta(\omega) L / l}
$$

We first compare the simulation result for propagation of an SFQ pulse to the analytical solution using Eqns. 1 and 2 as shown in Fig. 3. The SFQ pulse is approximated by a Gaussian with $V_{\text {in }}(t)=V_{0} e^{-t^{2} / 2 \tau^{2}}$, where the pulse magnitude $V_{0}$ of $1 \mathrm{mV}$ and FWHM $\tau$ of 1.88 ps produce $1 \Phi_{0}$. The simulation and numerical results agree, and both show significant dispersion at the longer lengths.

Fig. 4 shows pulse propagation in microstrips of length 8-64 $\mathrm{mm}$ as a function of width in the range $0.25-1.6 \mu \mathrm{m}$. Pulse amplitude decreases with length and is half the initial value after propagating $64 \mathrm{~mm}$. The narrower, higher impedance lines have more attentuation and dispersion due to increased kinetic inductance and the dominant contribution of metalic loss. For a given impedance, longer-range propagation can be achieved using a wider line and thicker dielectric.

\section{CIRCUIT DESIGN AND MEASUREMENT}

To verify our model we designed test circuits with different PTL lengths and two different PTL drivers that generated SFQ and double-flux-quantum (DFQ) pulses. Each PTL link consists of a driver, a $20 \Omega$ PTL stripline, and a receiver. The driver is fed with a differential input transformer [7] and Josephson Transmission Line (JTL) chain, and the receiver connects to a $2 \mathrm{mV}$ differential amplifier at the output. Circuits are powered using a resonant clock network at $3.5 \mathrm{GHz}$ that provisions the active area of the chip [27].

PTL links were characterized with pulses having two different spectral bandwidths, generated by two different a)
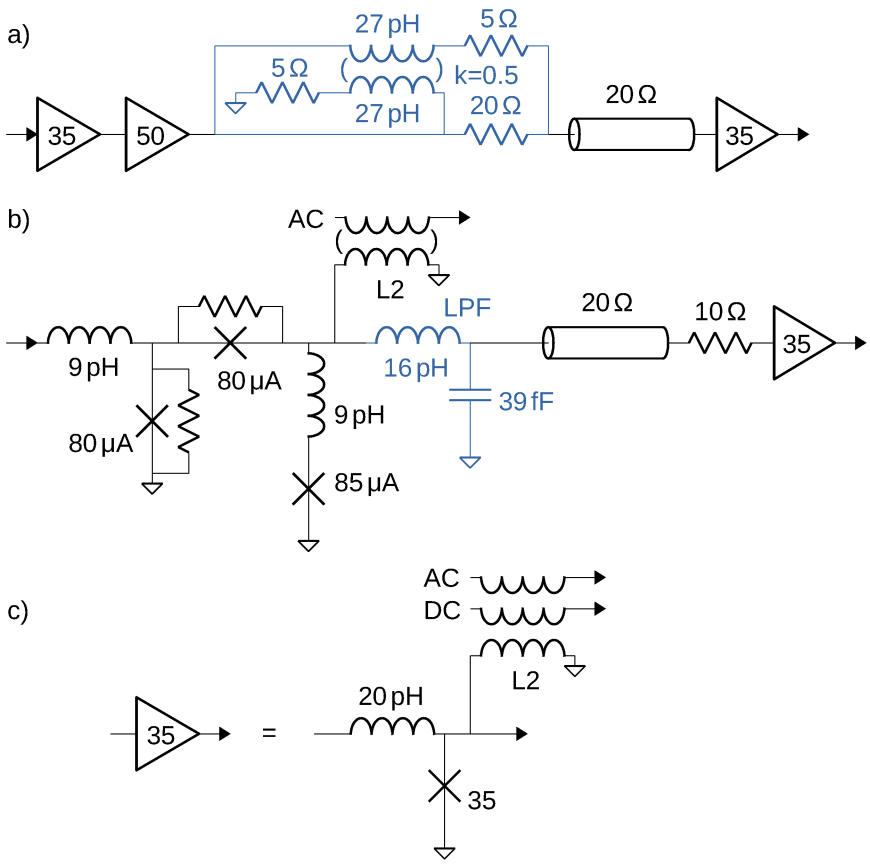

FIG. 5. Interconnect schematics. a) The SFQ driver launchs a full SFQ pulse onto the transmission line through a network that both steps up the voltage and provides source termination. b) The DFQ driver launches a pulse that is about the same peak amplitude, but twice as wide, as the SFQ. A lowpass filter (LPF) shapes the pulse. The receiver absorbs half of the signal - one SFQ - while the other half is resistively terminated. c) The standard JTL for a $35 \mu \mathrm{A}$ junction. The leading inductor has a reduced value of $10 \mathrm{pH}$ in the receiver JTL.

PTL drivers. The DFQ driver produces a pulse with twice the width of the SFQ, and similar peak amplitude. Due to the quadratic dependence of attenuation constant vs. frequency discussed above, the DFQ pulse is expected to propagate four times the distance. Schematics of the drivers are shown in Fig. 5.

The designs used effective terminations of $5-10 \Omega$ to 

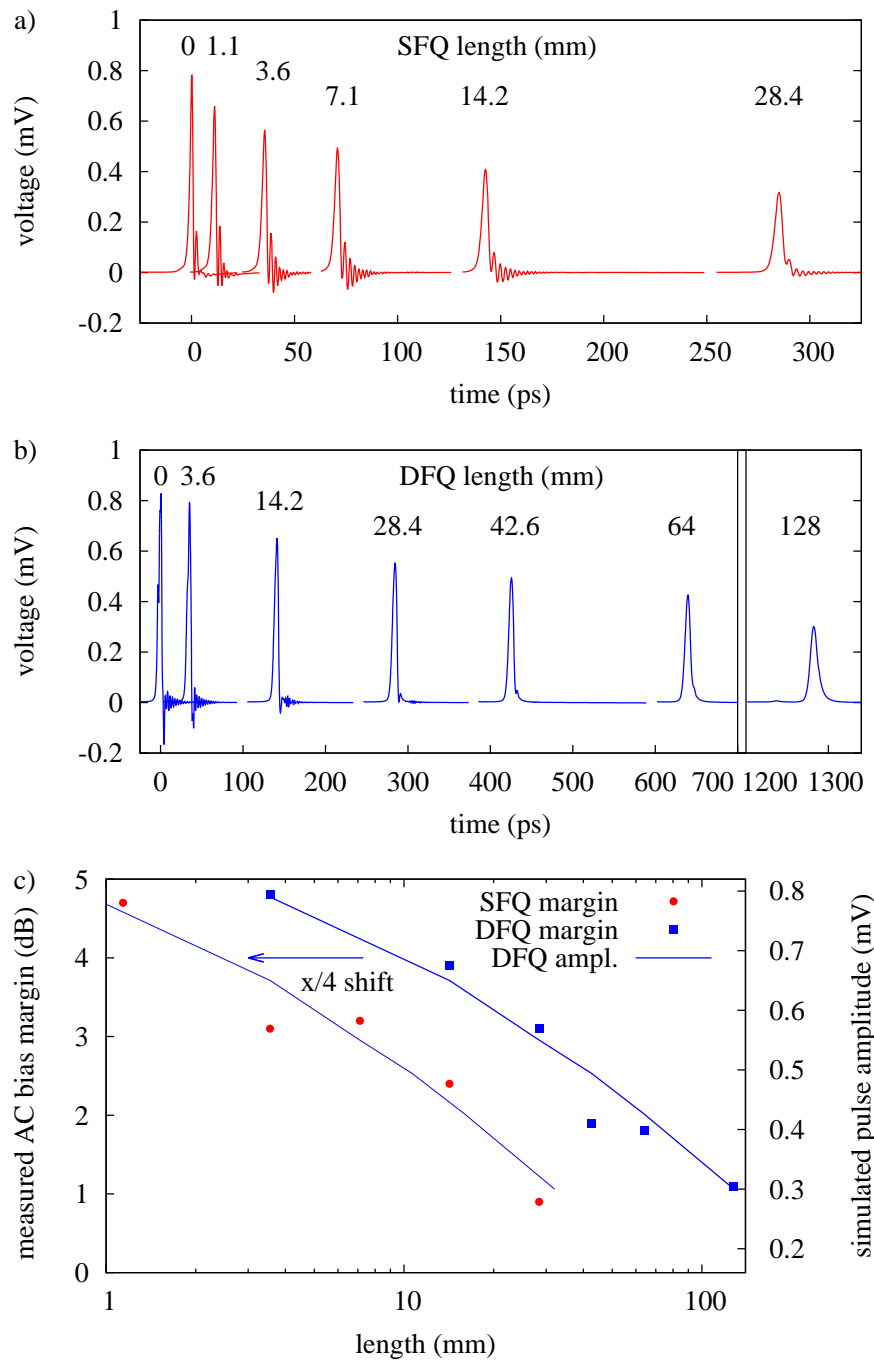

FIG. 6. Time domain Spectre simulations of a) SFQ and b) DFQ pulse propagation for various transmission line lengths. The earliest waveform is taken at the driver. Subsequent waveforms are taken at the end of resistevely terminated PTLs of given lengths. c) Measured AC bias margin (points) for the various SFQ and DFQ transmission line lengths using a "11110000111000110010" chirp pattern at $3.5 \mathrm{GHz}$, with a signal-averaged output waveform. Peak pulse amplitude read from the DFQ simulations are overlaid with the measured margins, using a linear fit given by the scale and offset on the right. The same curve is shown shifted to the left by a factor of $1 / 4$.

damp reflections. The SFQ driver used source termination. If the driver JTL were connected directly to a series resistor, only a fraction of an SFQ pulse would be launched on the PTL. The transformer between the JTL and the PTL steps up the voltage and launches a full SFQ. The $20 \Omega$ resistor bypasses the transformer to damp high frequencies.

The DFQ driver produces two SFQ pulses, as described in [1]. In the current implementation, the output terminal connects the central node of the driver, and the out- put signal averages over the voltages produced among the junctions. The two SFQ pulses partially overlap, and a simple LC low-pass filter on the output produces a suitably unitary waveform.

The $20 \Omega$ PTL is matched to the characteristic impedance of the $35 \mu \mathrm{A}$ receiver junction with critical current density $100 \mu \mathrm{A} / \mu \mathrm{m}^{2}$. The receiver junction absorbs an SFQ pulse when it triggers. For the DFQ design, this corresponds to only half the signal, and the other half is terminated in the $10 \Omega$ resistor 28 .

Hitting the timing window of the AC-powered receiver is an important design constraint. As the resonant clock network provides a global timing reference, the length of the PTL in each circuit is an integer number of $\lambda / 4$ segments, where $\lambda$ is the wavelength of the clock frequency. With appropriate phase selection at driver and reciever, the arrival time at the receiver is centered in the window for all PTL lengths.

The circuits were simulated in the time-domain with PTL models generated for the specific geometry used, described below. Simulations showed no degradation of margins due to reflections, at all lengths. The design margin for AC power is $5 \mathrm{~dB}( \pm 28 \%)$ for the short PTLs where attenuation and dispersion are small. The circuits included PTLs long enough to show margin degradation. The simulated dispersion of the data pulses as a function of PTL length is shown in Fig. 6a and b. These simulations also show four times longer propagation distance for the DFQ pulses, based on a comparison of peak amplitude at the longer lengths.

The circuits were fabricated in a process supplied by D-Wave [29], with six metal layers including the ground plane, Josephson junctions with $100 \mu \mathrm{A} / \mu \mathrm{m}^{2}$ critical current density, and with $0.25 \mu \mathrm{m}$ minimum feature size. The PTLs are implemented as stripline, with the signal layer between two ground layers connected with sidewall vias. The $20 \Omega$ line was optimized in HFSS using the design-rule metal and dielectric thicknesses of $200 \mathrm{~nm}$ and $\lambda_{L}$ of $100 \mathrm{~nm}$. The resulting geometry is a $0.950 \mu \mathrm{m}$ wire width and $0.675 \mu \mathrm{m}$ spacing to the via wall. PTL design is supported by the process design kit (PDK) environment using the Multi-Part-Path (MPP) parameteric cell in Cadence Virtuoso, and verification of connectivity and length using Cadence Assura layoutversus-schematic (LVS).

The speed of light in the PTL was verified using Sparameter measurements of a $\lambda / 2$ resonator on the same chip and using the same design as the PTLs of the functional circuits. The measured velocity of $99.5 \mu \mathrm{m} / \mathrm{ps}$ is within $3 \%$ of the design value, indicating sufficent fabrication process targeting.

Fig. 6r shows the measured AC bias margin as a function of PTL length for both SFQ and DFQ pulses. AC bias provides a measure of pulse amplitude at the receiver by changing receiver sensitivity. This global AC bias also influences the amplitude of the pulse generated by the driver, but this is a smaller effect due to SFQ pulse quantization. The measured $\mathrm{AC}$ margin for $\mathrm{DFQ}$ 
pulses is overlayed with simulated DFQ pulse height using data from Fig. 6b with a fitted scale and offset. The same DFQ pulse height curve, shifted to the left by a factor of $1 / 4$, overlays the SFQ data. This confirms the expected quadratic dependence of propagation distance on pulse width and the correctness of the design and simulation methodology. Both drivers show reduction of bias margin for longer PTLs. For practical purposes the accepatble length of the PTL is defined by the AC bias margin greater than $3 \mathrm{~dB}$ that corresponds to the PTL lengths of $7 \mathrm{~mm}$ and $28 \mathrm{~mm}$ for the two drivers.

\section{CONCLUSION}

Superconducting interconnects of arbitrary geometry and length can be simulated in HFSS using the MattisBardeen model. We have introduced a method to simulate propagation of ps pulses over supercondutcing PTL interconnects, based on HFSS simulations that provide an S-parameter model for time-domain simulations in Spectre. Using this method we have shown that the propagation distance is constrained by the high frequency components of the pulse spectrum and by the PTL geometry.

We have shown in simulation and test that $20 \Omega \mathrm{Nb}-$ based PTL interconnect with on-chip dielectric thickness of $200 \mathrm{~nm}$ can cover a distance of $7 \mathrm{~mm}$ using the SFQ driver and $28 \mathrm{~mm}$ using DFQ. Range scales with the square of the pulse width. The thicker dielectric associated with multichip packaging, and increased pulse width corresponding to $10 \mathrm{SFQ}$ pulses per bit, would extend the reach to meters.

\section{APPENDIX}

The $A B C D$ matrix for a lossy PTL can be found in 30]

$$
A B C D=\left[\begin{array}{cc}
\cosh (\gamma l) & Z_{0} \sinh (\gamma l) \\
\sinh (\gamma l) / Z_{0} & \cosh (\gamma l)
\end{array}\right]
$$

where $\gamma$ is the PTL propagation constant and $Z_{0}$ is the PTL characteristic impedance. Converting this into a scattering matrix [31] yields

$$
\left\{\begin{array}{l}
S_{11}=S_{22}=\frac{\left(Z_{0}^{2}-Z_{0 p}^{2}\right) \sinh (\gamma l)}{2 Z_{0} Z_{0 p} \cosh (\gamma l)+\left(Z_{0}^{2}-Z_{0 p}^{2}\right) \sinh (\gamma l)} \\
S_{21}=S_{12}=\frac{2 Z_{0} Z_{0 p}}{2 Z_{0} Z_{0 p} \cosh (\gamma l)+\left(Z_{0}^{2}-Z_{0 p}^{2}\right) \sinh (\gamma l)}
\end{array}\right.
$$

where $Z_{0 p}$ is the characteristic impedance of the PEC port. Substituting $\cosh (\gamma l)=\left(e^{\gamma l}+e^{-\gamma l}\right) / 2$ and $\sinh (\gamma l)=\left(e^{\gamma l}-e^{-\gamma l}\right) / 2$ into Eqns. 4 and solving for $e^{-\gamma l}$ and $Z_{0}$ leads to an expression for PTL characteristic impedance in terms of the S-parameters

and also leads to Eqn. 3

$$
Z_{0}=\mp Z_{0 p} \frac{\sqrt{\left(1-S_{11}^{2}+S_{21}^{2}\right)^{2}-4 S_{21}^{2}}}{\left(1-S_{11}\right)^{2}-S_{21}^{2}}
$$

\section{ACKNOWLEDGMENTS}

The authors acknowledge Yamil Morales and Tim Crowley for assisting with circuit test, Paul Chang for assisting with data reduction and analysis, and Joshua Strong for valuable discussions and help with HFSS simulations.
[1] Q. P. Herr, A. D. Smith, and M. S. Wire, Applied Physics Letters 80, 3210 (2002).

[2] Y. Hashimoto, S. Yorozu, T. Satoh, and T. Miyazaki, Applied Physics Letters 87, 022502 (2005).

[3] T. Filippov, D. Amparo, M. Kamkar, J. Walter, A. Kirichenko, O. Mukhanov, and I. Vernik, in 2017 16th International Superconductive Electronics Conference (ISEC) (IEEE, 2017), pp. 1-4.

[4] R. Farjadrad, M. Kuemerle, and B. Vinnakota, IEEE Micro 40, 15 (2019).

[5] D. A. Miller, Journal of Lightwave Technology 35, 346 (2017).

[6] J. Egan and et. al., to be published (2021).

[7] Q. P. Herr, A. Y. Herr, O. T. Oberg, and A. G. Ioannidis, Journal of Applied Physics 109, 103903 (2011).

[8] M. H. Volkmann, I. V. Vernik, and O. A. Mukhanov, IEEE Transactions on Applied Superconductivity 25, 1 (2015).

[9] F. China, N. Tsuji, T. Narama, N. Takeuchi, T. Ortlepp, Y. Yamanashi, and N. Yoshikawa, IEEE Transactions on Applied Superconductivity 27, 1 (2016).
[10] M. Rafique, I. Kataeva, H. Engseth, M. Tarasov, and A. Kidiyarova-Shevchenko, Superconductor Science and Technology 18, 1065 (2005).

[11] N. Takeuchi, Y. Yamanashi, Y. Saito, and N. Yoshikawa, Physica C: Superconductivity 469, 1662 (2009).

[12] S. V. Polonsky, V. K. Semenov, and D. F. Schneider, IEEE Transactions on Applied Superconductivity 3, 2598 (1993).

[13] K. Takagi, M. Tanaka, S. Iwasaki, R. Kasagi, I. Kataeva, S. Nagasawa, T. Satoh, H. Akaike, and A. Fujimaki, IEEE Transactions on Applied Superconductivity 19, 617 (2009).

[14] R. N. Das, V. Bolkhovsky, S. K. Tolpygo, P. Gouker, L. M. Johnson, E. A. Dauler, and M. A. Gouker, in 2017 IEEE 67th Electronic Components and Technology Conference (ECTC) (2017), pp. 675-683.

[15] A. Shukla, B. Chonigman, A. Sahu, D. Kirichenko, A. Inamdar, and D. Gupta, IEEE Transactions on Applied Superconductivity 29, 1 (2019).

[16] D. Mattis and J. Bardeen, Physical Review 111, 412 (1958). 
[17] R. L. Kautz, Journal of Applied Physics 49, 308 (1978).

[18] R. Peterson and D. McDonald, IEEE Transactions on Magnetics 13, 887 (1977).

[19] C. Chi, W. Gallagher, I. Duling, D. Grischkowsky, N. Halas, M. Ketchen, and A. Kleinsasser, IEEE Transactions on Magnetics 23, 1666 (1987).

[20] $3 D$ electromagnetic field simulator for $R F$ and wireless design, https://www.ansys.com/products/electronics/ansyshfss.

[21] Y. Shlepnev, in DesignCon IBIS Summit (2010).

[22] L. A. Vainshtein, Soviet Physics Uspekhi 19, 189 (1976).

[23] P. Triverio, S. Grivet-Talocia, M. S. Nakhla, F. G. Canavero, and R. Achar, IEEE Transactions on Advanced Packaging 30, 795 (2007).

[24] V. V. Talanov, L. V. Mercaldo, and S. M. Anlage, Review of Scientific Instruments 71, 2136 (2000).
[25] N. Klein, H. Chaloupka, G. Müller, S. Orbach, H. Piel, B. Roas, L. Schultz, U. Klein, and M. Peiniger, Journal of Applied Physics 67, 6940 (1990).

[26] D. E. Oates, S. K. Tolpygo, and V. Bolkhovsky, IEEE Transactions on Applied Superconductivity 27, 1 (2017).

[27] V. V. Talanov and J. A. Strong, Clock distribution network for a superconducting integrated circuit (2017), US Patent 9,722,589.

[28] Q. P. Herr, M. S. Wire, and A. D. Smith, IEEE transactions on Applied Superconductivity 13, 463 (2003).

[29] A. Berkley, M. Johnson, P. Bunyk, R. Harris, J. Johansson, T. Lanting, E. Ladizinsky, E. Tolkacheva, M. Amin, and G. Rose, Superconductor Science and Technology 23, 105014 (2010).

[30] C. R. Paul, Analysis of multiconductor transmission lines (John Wiley \& Sons, 2007), chap. 6.

[31] D. M. Pozar, Microwave engineering 4th edition (John Wiley \& Sons, 2011), chap. 4. 